\title{
Stability of Stevioside and Glucosyl-Stevioside under Acidic Conditions and its Degradation Products
}

\author{
Abubakr Musa $^{1,2}$, Ming Miao ${ }^{1}$, Mohammed A.A Gasmalla ${ }^{1,3}$, Tao Zhang ${ }^{1}$, Ahmed Eibaid ${ }^{1}$, Waleed Aboshora ${ }^{1}$, \\ Bo Jiang, ${ }^{1, *}$ \\ ${ }^{1}$ State Key Laboratory of Food Science and Technology, School of Food Science and Technology, Jiangnan University, 1800 Lihu \\ Ave., Wuxi, Jiangsu, China \\ ${ }^{2}$ Sugar Institute, University of Gezira,Wad Madani, Al Gezira, Sudan \\ ${ }^{3}$ Department of Nutrition \& Food Technology, Faculty of Science and Technology, Omdurman Islamic University, Khartoum, Sudan \\ *Corresponding author: bjiang@jiangnan.edu.cn
}

Received April 30, 2014; Revised May 12, 2014; Accepted May 14, 2014

\begin{abstract}
The stability of stevioside and mono- and di-glucosyl-stevioside(produced via Leuconostoccitreum SK24.002 alternansucrase acceptor reaction), and the possible formation of the steviol at elevated temperature and different $\mathrm{pH}$ levels was assessed, covering a typical $\mathrm{pH}$ range that simulated both relevant and extreme beverage storage conditions. Acid solutions mixed with stevioside or mono- and di-glucosyl- stevioside after 24, 48, and $72 \mathrm{~h}$ of storage time at 50 and $80^{\circ} \mathrm{C}$ were analysed. Under mild conditions (at a pH range of $2-6.5$ over $72 \mathrm{~h}$ and $50^{\circ} \mathrm{C}$ ) stevioside and mono- and di-glucosyl-stevioside showed good stability. Degradation of up to $55 \%$ was observed at $\mathrm{pH} 3$ and $80^{\circ} \mathrm{C}$ after $72 \mathrm{~h}$, and stevioside was less stable than mono-glucosyl-stevioside. Complete degradation was observed at $\mathrm{pH} 2$ and $80^{\circ} \mathrm{C}$ after $72 \mathrm{~h}$. Stevioside and mono- and di-glucosyl-stevioside and their degradation products were analysed by high-performance liquid chromatography with a diode array detector (DAD-HPLC) on an NH2 analytical column, and the identity of the degradation products was confirmed by liquid chromatographyelectrospray ionization mass spectrometry (LC-ESI-MS) in negative mode. Practical Application: Utilizing transglucosylated stevioside products as natural sweeteners or sweetness enhancers, impose to understand physicochemical profiles of transglucosylated stevioside products in various systems of interest, here we studied the stability of a steviosid and transglucosylated stevioside products at elevated temperature and different $\mathrm{pH}$ levels covering a typical pH range that emulated both relevant and extreme beverage storage conditions.
\end{abstract}

Keywords: stevioside, mono- and di-glucosyl-stevioside, stability, degradation products, LC-MS analysis

Cite This Article: Abubakr Musa, Ming Miao, Mohammed A.A Gasmalla, TaoZhang, Ahmed Eibaid, Waleed Aboshora, and Bo Jiang, "Stability of Stevioside and Glucosyl-Stevioside under Acidic Conditions and its Degradation Products." Journal of Food and Nutrition Research, vol. 2, no. 4 (2014): 198-203. doi: 10.12691/jfnr-2-4-11.

\section{Introduction}

Because of awareness of the obesity problem, many food manufacturers are trying to reduce calories by introducing synthetic and natural noncaloric sweeteners into their systems. Stevioside, a high-intensity nonnutritive sweetener was extracted from the leaves of Stevia rebaudiana Bertoni, a plant native to north eastern Paraguay, it is a white, crystalline and odorless powder, approximately 300 times sweeter than sucrose [1] is one such example in recent years, which resulted in the isolation of several potently sweet diterpenoid glycosides namely rebaudiosides $\mathrm{A}$ and $\mathrm{D}$, stevioside, and dulcoside A, also known as stevia sweeteners of which stevioside and rebaudioside A are the major ones. These compounds are all glycosides of the diterpene ent-13-hydroxykaur-16en-19-oic acid known as steviol [2,3].

Steivolglucosylis noncaloric because of the sugar units in their structuerwere connected to each other and to the steviol scaffold by $\beta$-glycosidic bonds (or a $\beta$-glycosidic ester bond at carbon 19). As a consequence, the human digestive tract is unable to break down steivolglucosyl [4]. The microbial flora of the colon will partly degrade the steivolglucosyl to steviol. After being taken up by the gut, the steviol then is transported to the liver by the portal vein and converted to steviolglucuronide, which is excreted in the urine [5].

Investigation about the stability of steivolglucosyl in different foodstuffs are quite fragmentary and rare and deal for the most part with the use of steivolglucosyl in soft drinks [6], compared the stability of stevioside and rebaudioside A in soft drinks. They found that the rebaudioside A is more stable than stevioside. And degradation of stevioside up to $70 \%$ was observed after 72 h of storage at $80^{\circ} \mathrm{C}$. The stability of the steivolglucosyl increased with increasing $\mathrm{pH}$, which is consistent with an acid-catalysed hydrolysis of glucose units[7].

Apart from isolating transglucosylated stevioside products and utilising them as possible natural sweeteners or sweetness enhancers, we have recently separated two main transglucosylated stevioside products produced 
during biotransformation of stevioside by leuconostoccitreum SK 24.002 alternansucrase acceptor reaction [8]. It is important to understand the physicochemical profiles of thetransglucosylated stevioside products in various systems of interest. This article describes the stability of a steviosid, mono- and didlucosyl- stevioside at elevated temperature and different $\mathrm{pH}$ levels. The mono- and di-dlucosyl- stevioside used in this study were $13-\{[\alpha-D$-glucopyranosyl- $(1 \rightarrow 6)-\beta-D-$ glucopyranosyl-( $1 \rightarrow 2)-\beta$-D-glucopyranosyl]oxy $\}$ kaur-16en-19-oic acid $\beta$-D glucopyranosyl eater and $13-\{[\alpha-D-$ glucopyranosyl-( $1 \rightarrow 3)$ - $\alpha$-D-glucopyranosyl- $(1 \rightarrow 6)-\beta$-Dglucopyranosyl-( $1 \rightarrow 2)-\beta$-D-glucopyranosyl]oxy $\}$ kaur-16en-19-oic acid $\beta$-D glucopyranosyl eater, respectively (see Figure 1).
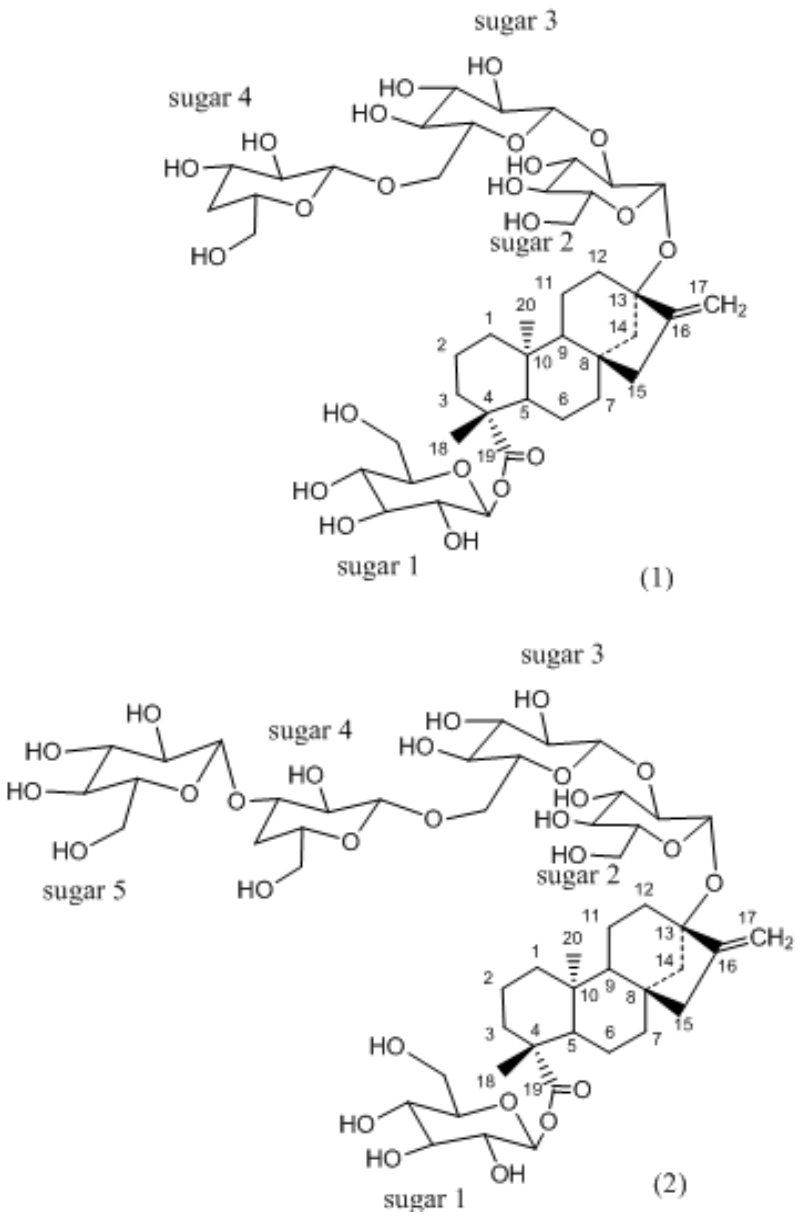

Figure 1. Structure of mono-glucosyl-stevioside (1) and di-glucosylstevioside (2)

\section{Materials and Methods}

\subsection{Materials}

Stevioside (purity 99\%) was purchased from Wako Pure Chemical Industries, Ltd., Japan. Stevioside (purity $>80 \%$ ) was purchased from Aladdin Reagent Database Inc., Shanghai, China. All other chemicals were of analytical grade and were obtained from Sinopharm Chemical Reagent Co., Ltd., Shanghai, China. Transglucosylated stevioside products were produced as reported previously [8], and were separated themon- and di- glucosyl-stevioside by using macropours resin AB-8 flowed by semi-preparative HPLC.

\subsection{Sample Preparation}

The degradation of the steviol glycosides was studied in acidic medium. $5 \mathrm{mg}$ of stevioside, mono- and diglucosyl-steviosidesamples were added to $10 \mathrm{ml}$ tubes containing roughly $6 \mathrm{~m}$ Lofacid solutions in the $\mathrm{pH}$ range of (2 - 6.5), prepared from concentrated HCL aqueous solutions. $0.5 \mathrm{~mL}$ of the sample solutions was transferred to a $1.5 \mathrm{~mL}$ tube. The $1.5 \mathrm{~mL}$ tubes were stored for up to $72 \mathrm{~h}$ in a water bath at 50 and $80^{\circ} \mathrm{C}$. After $0,24,48$, and $72 \mathrm{~h}, 1.5 \mathrm{~mL}$ tube of each sample was removed for further analysis. Losses dueto evaporation were compensated by the addition of water. The samples were subjected to HPLC using an NH2 analytical column and UV detection, and the concentration was determined using a standard calibration curve of stevioside standard solution. These degradation experiments were performed in duplicate for Stevioside, mono- and di-glucosyl-stevioside, respectively.

\subsection{Analysis Procedures (HPLC\& LC.MS)}

Stevioside, mono- and di- glucosyl-stevioside and their degradation products were analysed on an Agilent 1200 series HPLC system (Agilent Technologies, USA). Analysis was performed, using NH2 column (Shodex Asahipak, NH2P-50 4E, ID 4.6*250 mm, $5 \mu \mathrm{m}$, Showa Denko K. K, Tokyo, Japan). The mobile phase consisted of acetonitrile/water (7.5:2.5 v/v) and was delivered with a flow rate of $1.0 \mathrm{~mL} / \mathrm{min}$ at $30^{\circ} \mathrm{C}$. The injection volume was $30 \mu \mathrm{L}$. The diode array detector was set to a wavelength of $210 \mathrm{~nm}$. LC.MS/MS (Waters Acquity UPLC and PDA; Waters MaldiSynapt Q-T of MS) was operated in a negative ion detection mode; Ultra pure synthetic air was used as nebulisationde solvation gas (flow rate $=500 \mathrm{~L} / \mathrm{h}$ ) and MS fragment ions were obtained with $20 \mathrm{eV}$ collision energy. A mixture of acetonitrile and water was used as the eluent, gradient from 75: $25 \mathrm{v} / \mathrm{v}$ (2 $\mathrm{min}$ ) to $50: 50 \mathrm{v} / \mathrm{v}(30 \mathrm{~min})$, the flow rate was $0.8 \mathrm{ml} / \mathrm{min}$.

\section{Results and Discussion}

\subsection{Stability of Stevioside and Mono- and Di- glucosyl Steviosidein Acid Solution}

The main objective of this study was to assess the stability of stevioside and mono- and diglucosylstevioside under a variety of conditions covering a typical pH range of beverage processing. We conducted an accelerated shelf-life studywith severe storage condition (50 and $80^{\circ} \mathrm{C}$ ) to induce a thermal breakdown of the stevioside and mono- and di-glucosylstevioside.

Figure 2, illustrate the stability of stevioside and monoand di-glucosyl-seviosideinaqueous acid solutions at $50^{\circ} \mathrm{C}$. In aqueous acid solution stevioside and mono- and diglucosyl-sevioside are remarkably stable over a wide range of $\mathrm{pH}$ and temperature. Under thermal treatment in a $\mathrm{pH}$ range of 2-6.5 over $72 \mathrm{~h}$ empirically, no significant degradation of stevioside and mono- and di-glucosylsevioside could be observed at $50^{\circ} \mathrm{C}$. This was in good agreement with Gerhard Kroyer [9], who found that, no degradation of stevioside in a $\mathrm{pH}$ range 2-10 after $2 \mathrm{~h}$ at $60^{\circ} \mathrm{C}$. As shown in Figure 3, degradation of 35, 24 and $54 \%$ for stevioside, mono- and di-glucosyl-sevioside, respectively were occurringat $\mathrm{pH} 3$ by heating at a 
temperature of $80^{\circ} \mathrm{C}$ for $72 \mathrm{~h}$ storage. Which indicated that mono-glucosyl-stevioside was more stable than stevioside and di-glucosyl-sevioside. And confirmed that the mono-glucosyl-stevioside have the same characteristic of rebaudioside A, as reported previously, rebaudioside A was more stable against acid hydrolysis thanstevioside [6], also Chang reported a $36 \%$ oss in stevioside concentration in beverages after 4 months at $37^{\circ} \mathrm{C}$, whereas rebaudioside A was degraded to only $25 \%$ [10].
However strong acidic conditions ( $\mathrm{pH} \mathrm{2}$ ) accelerated the decomposition of stevioside, mono- and di-glucosylsevioside, resulting in total decomposition after incubation at a temperature of $80^{\circ} \mathrm{C}$ for $72 \mathrm{~h}$ of storage.Similar results were reported by Kroyer [11], who observed a total decomposition of stevioside at low $\mathrm{pH}$.

From above results, obvious that the stability of the stevioside, mono- and di-glucosyl-sevioside were $\mathrm{pH}$, temperature, and time dependents.
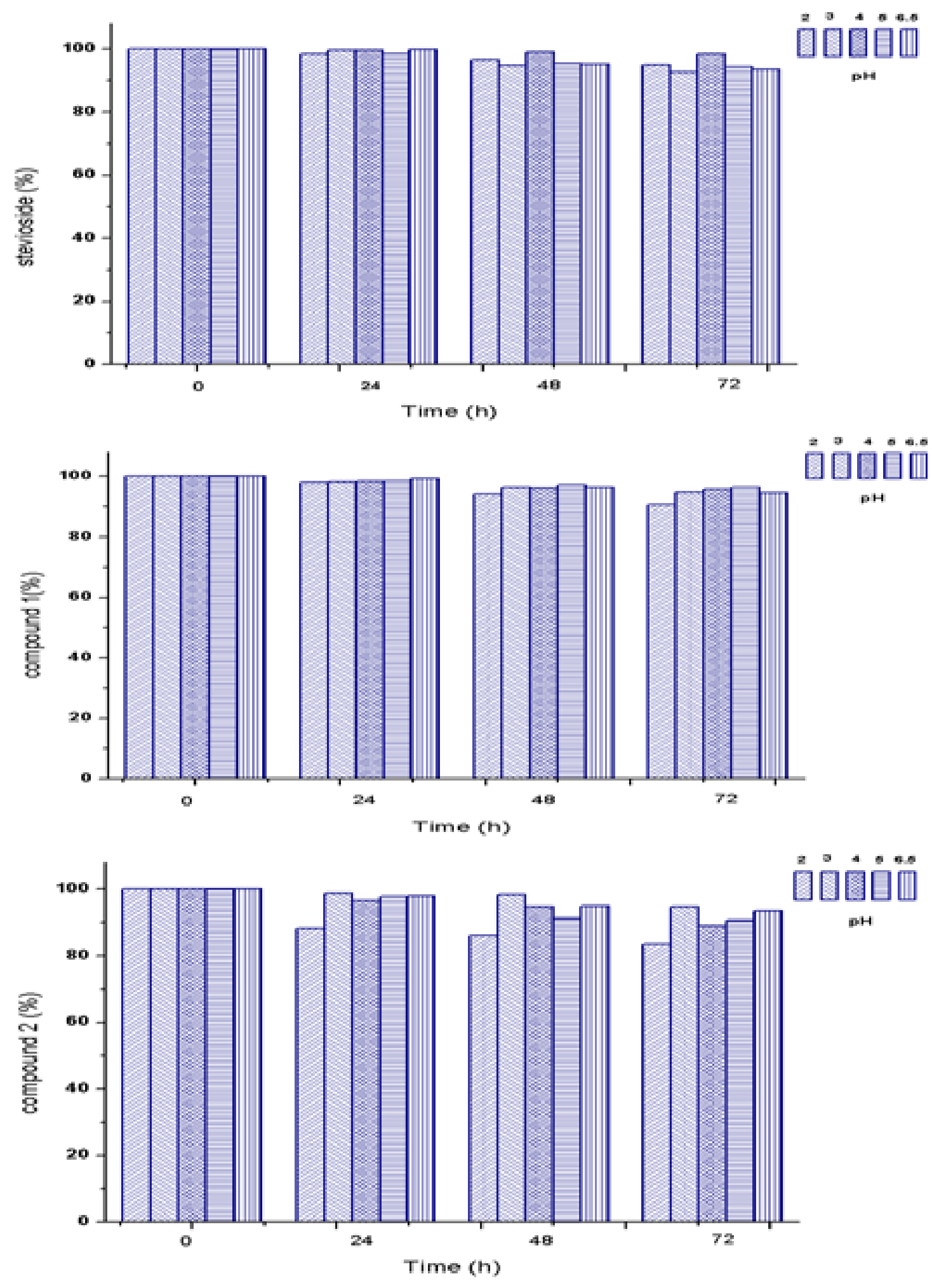

Figure 2. Stability of stevioside and mono- and di-glucosyl-sevioside (compounds $1 \& 2$ ) at $50^{\circ} \mathrm{C}$ 

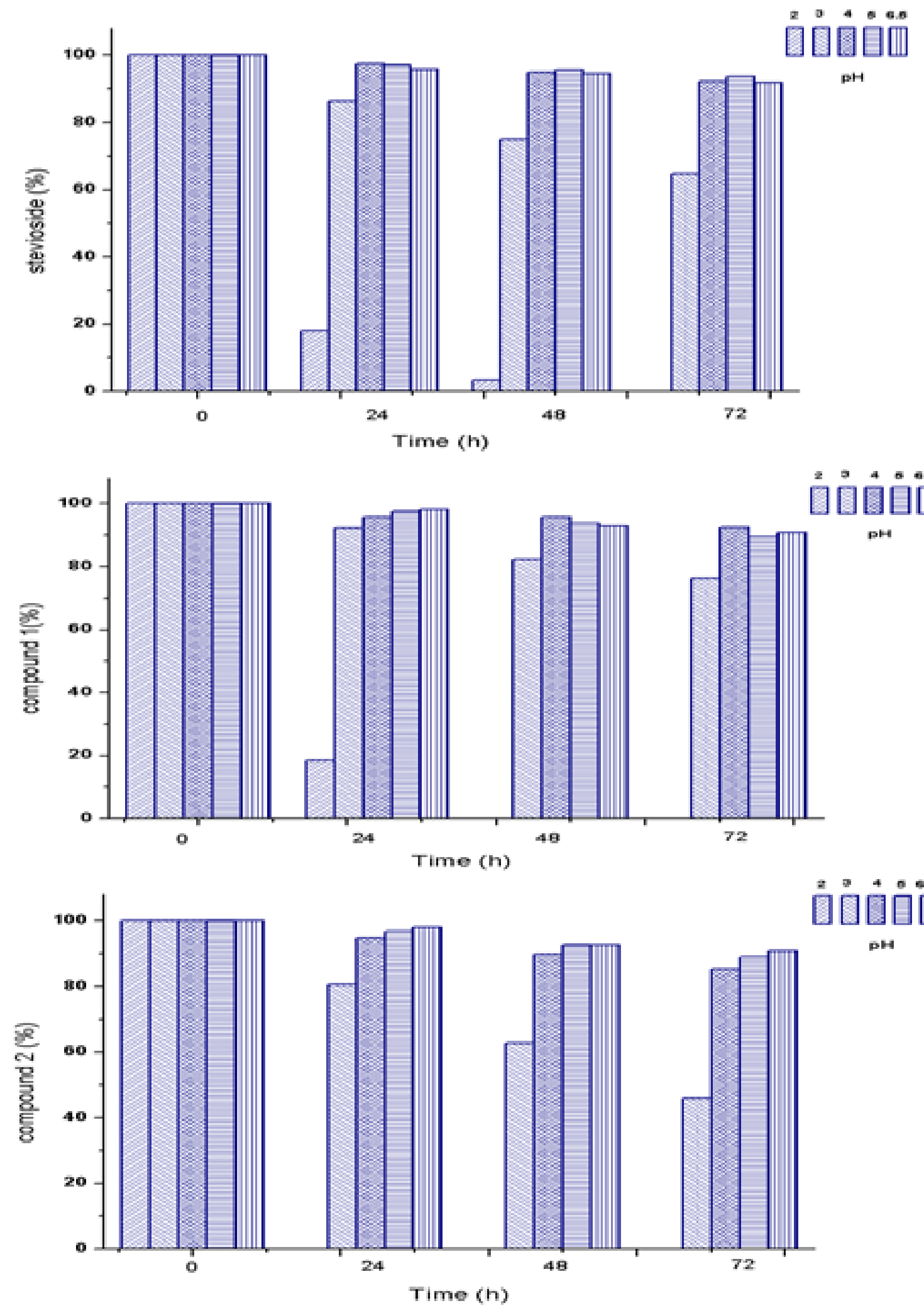

Figure 3. Stability of stevioside and mono- and di-glucosyl-sevioside (compounds $1 \& 2$ ) at $80^{\circ} \mathrm{C}$

\subsection{Degradation Products of Stevioside and Mono- and Di-glucosyl Stevioside}

Figure 4, represent the total ion chromatograms of degradation products of stevioside, compound 1 and compound 2 (mono- and di-glucosyl-sevioside), indicated that the mono-glucosyl-stevioside was more stable and less formation of stivol at low $\mathrm{pH}$ (2), and high temperature over $72 \mathrm{~h}$ storage, comparing with steviside and di-glucosyl-stevioside at the same condition. Also, we are herewith reporting the mass ion of the stevioside, mono- and di-glucosyl-sevioside and its major degradation products obtained during course of study by using ESI-MS in negative ion mode to further characterize the degradation products because the analyte signals should be about 10 times higher than in positive mode $[12,13]$. Table 1 indicates the molecular ions of the most identified degradation products, whereas in MS/MS fragmentation these compounds were readily confirmed 
through subsequent glycosidic losses of fragments of 162 Da. The degradation pattern of stivioside in acidic solution were investigated. Besides the molecular ion for stevioside (m/z 803), themass ( $\mathrm{m} / \mathrm{z}$ 641) representing steviolbioside and rubusoside were appeared at retention time 5.3 and 6.7, additionally, steviolmonoside ( $\mathrm{m} / \mathrm{z} 479$ ) was detected at 7.6 retention time. And the peak corresponding to steviol (m/z 317) was detected in the MS spectra within the 8.2 min retention time. This results in agreements with Wölwer-Rieck [6], who reported that the the molecular ions of $(803,641,479$ and $317 \mathrm{~m} / \mathrm{z})$ were assigned tostevioside, steviolbioside orrubusoside, steviolmonoside and steviolrecpectivly. Also our finding are consistent withthe results reported by Rodrigo R. Catharino [14], who observed that the hydrolysis of the stevioside molecule to steviolwas occurred in aqueous acidic solutions. The mass ion of the mono-glucosyl-stevioside and its degradation product showed, the presence of mono-glucosyl-stevioside (m/z 965) at retention time 4.7, stevioside $(\mathrm{m} / \mathrm{z} 803)$ at retention time 5.8 , the mass molecular ion of (m/z 641) corresponding to steviolbioside and rubusoside were detected at retention time 6.5 and 7.3, steviolmonoside ( $\mathrm{m} / \mathrm{z} 479)$ was appeared at retention time 6.3 and steviol $(\mathrm{m} / \mathrm{z} 317)$ was detected at 8.2 retention time. The investigation of degradation of diglucosyl-stevioside in aqueous acidic solutions revealed, the presences of di-glucosyl-stevioside (m/z 1127), monoglucosyl-stevioside (m/z 965), stevioside (m/z 803) andsteviol (m/z 317) at retention times 4.5, 5.5, 6.5 and 8.2 respectively. And the mass ( $\mathrm{m} / \mathrm{z} 641)$ corresponding to steviolbioside or rubusoside was detected at retention time 6.7.

Table 1. Molecular Ions of the Detected Steviol andSteviol Glycosides

\begin{tabular}{cccc}
\hline name & {$[\mathrm{M}-\mathrm{H}]^{-}$} & name & {$\left[\mathrm{M}-\mathrm{H}^{\mathrm{I}^{-}}\right.$} \\
steviol & 317 & stevioside & 803 \\
steviolmonoside & 479 & compound 1 & 965 \\
steviolbioside & 641 & compound 2 & 1127 \\
rubusoside & 641 & & \\
\hline
\end{tabular}

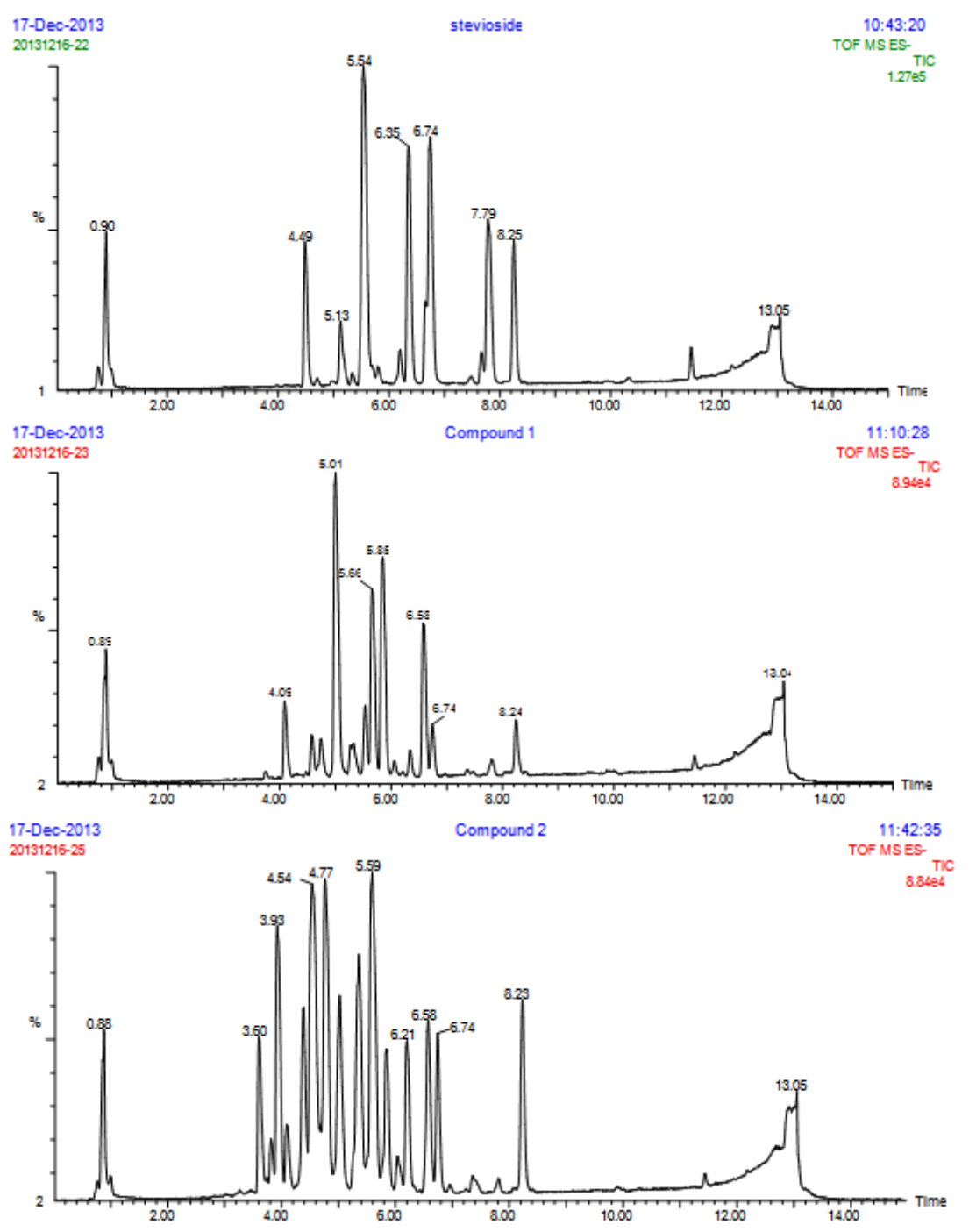

Figure 4. Total ion chromatograms of degradation products of stevioside, compound 1 and compound 2

\section{Conclusion}

The stability of stevioside and mono- and di-glucosylsteviosideinaqueous acidic solutions are $\mathrm{pH}$, temperature and time dependents. The rate and extent of degradation product formation was increased under acidic conditions (lower $\mathrm{pH}$ )and at higher temperatures with the formation of degradation products mainly occurring after extended period of storage. Generally, under mild conditions (in a $\mathrm{pH}$ range $2-6.5$ over $72 \mathrm{~h}$ and $50^{\circ} \mathrm{C}$ ) stevioside and monoand di-glucosyl-stevioside showed good stability. However, under extreme conditions at high temperatures 
of 80 and $\mathrm{pH} 3$ for 72 h of storage, a losses of up to 35, 24 and $54 \%$ of initial levels of stevioside, mono- and diglucosyl-sevioside, respectively were occurred. Whereas strong acidic conditions $(\mathrm{pH} 2)$ accelerated the decomposition of stevioside, mono- and di-glucosylsevioside, resulting in total decomposition after incubation at a temperature of $80^{\circ} \mathrm{C}$ for $72 \mathrm{~h}$. the results obtained from the total ion chromatograms, indicated that the mono-glucosyl-stevioside was more stable and less formation of stivol at low $\mathrm{pH}$ (2), and high temperature over 72 h of storage, comparing with stevioside and diglucosyl-stevioside at the same condition. Thus, the mono-glycosylated-stevioside can be used as sweetener in beverage industry with expecting of high stability and improving of the taste quality.

\section{Acknowledgment}

This research was financially supported by the National Natural Science Foundation of China (31000764, 20976073, 31230057), the National High Technology Research and Development Program of China (2013AA102102) and the Science \& Technology Pillar Program of Jiangsu Province (BE2013647, BE2012613, BY2012049).

\section{Reference}

[1] Soejarto, D.D., et al., Potential sweetening agents of plant origin. II. Field search for sweet-tastingStevia species. Economic Botany, 1983. 37 (1): p. 71-79.

[2] Brandle, J.E., A.N. Starratt, and M. Gijzen, Stevia rebaudiana: its agricultural, biological, and chemical properties. Canadian journal of plant science, 1998. 78 (4): p. 527-536.
[3] Steinmetz, W.E. and A. Lin, NMR studies of the conformation of the natural sweetener rebaudioside A. Carbohydrate research, 2009. 344 (18): p. 2533-2538.

[4] Geuns, J.M.C., et al., Metabolism of stevioside by healthy subjects. Experimental biology and medicine, 2007. 232 (1): p. 164-173.

[5] Geuns, J.M.C., et al., Identification of steviol glucuronide in human urine. Journal of agricultural and food chemistry, 2006. 54 (7): p. 2794-2798.

[6] Wölwer-Rieck, U., W. Tomberg, and A. Wawrzun, Investigations on the stability of stevioside and rebaudioside A in soft drinks. Journal of agricultural and food chemistry, 2010. 58 (23): p. 12216-12220.

[7] Jooken, E., et al., Stability of Steviol Glycosides in Several Food Matrices. Journal of agricultural and food chemistry, 2012. 60 (42): p. 10606-10612.

[8] Musa, A., et al., Biotransformation of stevioside by Leuconostoc citreum SK24. 002 alternansucrase acceptor reaction. Food chemistry, 2014. 146: p. 23-29.

[9] Kroyer, G., Stevioside and Stevia-sweetener in food: application, stability and interaction with food ingredients. Journal fü $\mathrm{r}$ Verbraucherschutz und Lebensmittelsicherheit, 2010. 5 (2): p. 225-229.

[10] Chang, S.S. and J.M. Cook, Stability studies of stevioside and rebaudioside A in carbonated beverages. Journal of agricultural and food chemistry, 1983. 31 (2): p. 409-412.

[11] Kroyer, G.T., The low calorie sweetener stevioside: stability and interaction with food ingredients. LWT-Food Science and Technology, 1999. 32 (8): p. 509-512.

[12] Choi, Y.H., et al., Supercritical fluid extraction and liquid chromatographic-electrospray mass spectrometric analysis of stevioside fromStevia rebaudiana leaves. Chromatographia, 2002. 55 (9-10): p. 617-620.

[13] Gardana, C., M. Scaglianti, and P. Simonetti, Evaluation of steviol and its glycosides in Stevia rebaudiana leaves and commercial sweetener by ultra-high-performance liquid chromatography-mass spectrometry. Journal of chromatography A, 2010. 1217 (9): p. 1463-1470.

[14] Catharino, R.R. and L.S. Santos, On-line monitoring of stevioside sweetener hydrolysis to steviol in acidic aqueous solutions. Food chemistry, 2012. 133 (4): p. 1632-1635. 